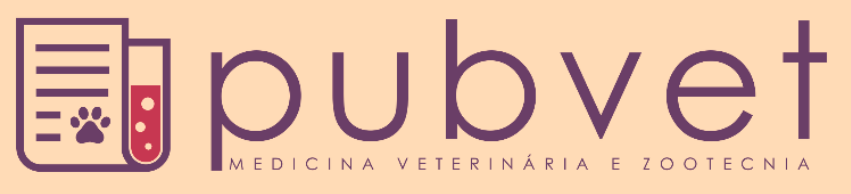

https://doi.org/10.31533/pubvet.v13n9a401.1-11

\title{
Resposta imune-inflamatória a agentes infecciosos microambiente hepático de cães e gatos: revisão
}

\author{
Társsila Mara Vieira Ferreira ${ }^{1} \bullet$, Diana Célia Sousa Nunes-Pinheiro ${ }^{2 *} \bullet$ \\ ${ }^{1}$ Doutoranda em Ciências Veterinárias pelo Programa de Pós-graduação em Ciências Veterinárias. Laboratório de Imunologia e Bioquímica \\ Animal. Faculdade de Veterinária. Universidade Estadual do Ceará. \\ ${ }^{2}$ Docente do Programa de Pós-graduação em Ciências Veterinárias e da Faculdade de Veterinária da Universidade Estadual do Ceará. \\ Av.Dr. Silas Munguba, 1700, Campus do Itaperi, Fortaleza-CE. CEP:60.714-903. \\ *Autor para correspondência e-mail: diana.pinheiro@uece.br
}

Resumo. O fígado é um órgão de alta exposição a antígenos circulantes, e seu ambiente é povoado por células imunes inatas que prontamente respondem a agentes invasores. Entretanto, uma resposta imune a agentes infecciosos pode desencadear um desequilíbrio nos mecanismos de tolerância, levando a um estado de estresse oxidativo, que favorece ao dano tecidual e pode provocar repercussões sistêmicas. Vale ressaltar que algumas doenças parasitárias como anaplasmose, babesiose, clonorquíase, dirofilariose, erliquiose, histoplasmose e platinossomíase podem provocar desregulação nos mecanismos de oxidação induzindo alterações hepáticas. Neste sentido, esta revisão aborda os mecanismos celulares da resposta imune hepática na homeostase e na progressão de distúrbios hepáticos que estão associados a imunopatogenia de algumas doenças parasitárias que afetam cães e gatos.

Palavras-chave: fígado, resposta imune hepática, estresse oxidativo, parasitas, cão e gato

\section{Immune-inflammatory response to infectious agents in the hepatic microenvironment of dogs and cats: a review}

\begin{abstract}
The liver is an organ of high exposure to circulating antigens, and its environment is populated by innate immune cells that readily respond to invading agents. However, an immune response to infectious agents might trigger an imbalance in the mechanisms of tolerance, leading to a state of oxidative stress, which favors tissue damage and might cause systemic repercussions. It is worth noting that some parasitic diseases such as anaplasmosis, babesiosis, clonorchiasis, heartworm disease, ehrlichiosis, histoplasmosis and platinossomiasis may cause dysregulation in oxidation mechanisms inducing hepatic alterations. In this sense, this review focuses on the cellular mechanisms of liver immune response in homeostasis and progression of hepatic disorders that are associated with immunopathogenesis of some parasitic diseases that affect dogs and cats.
\end{abstract}

Key words: liver, hepatic immune response, oxidative stress, parasites, dog and cat

\section{Respuesta inmune-inflamatoria a agentes infecciosos en el microambiente hepático de perros y gatos: revisión}

Resumen. El hígado es un órgano de alta exposición a antígenos circulantes, y su entorno está poblado por células inmunes innatas que responden fácilmente a los agentes invasores. Sin embargo, una respuesta inmune a los agentes infecciosos puede desencadenar un desequilibrio en los mecanismos de tolerancia, lo que lleva a un estado de estrés oxidativo, que favorece el daño tisular y puede causar repercusiones sistémicas. Cabe destacar que algunas enfermedades parasitarias como la anaplasmosis, babesiosis, clonorquiasis, 
enfermedad del gusano del corazón, erliquiosis, histoplasmosis y platinossomiasis pueden causar desregulación en los mecanismos de oxidación que inducen alteraciones hepáticas. En este sentido, esta revisión se centra en los mecanismos celulares de la respuesta inmune hepática en la homeostasis y la progresión de los trastornos hepáticos que están asociados con la inmunopatogenia de algunas enfermedades parasitarias que afectan a perros y gatos.

Palabras clave: hígado, respuesta inmune hepática, estrés oxidativo, parásitos, perros y gatos

\section{Introdução}

O fígado compreende o órgão metabólico central do organismo. Todavia, trata-se de um órgão importante da resposta imune inata em virtude da exposição regular a produtos dietéticos e da microbiota residente no intestino, bem como partículas antigênicas e patógenas circulantes pelo organismo (Mackness et al., 2010). Diante disso, ele apresenta uma ampla rede de células imune residentes, as quais produzem proteínas de fase aguda, componentes do complemento, citocinas e quimiocinas, desencadeando uma resposta imune complexa. O sistema imunológico hepático tolera moléculas inofensivas e, ao mesmo tempo, permanece alerta para possíveis agentes infecciosos (Nemeth et al., 2009). Todavia, a incapacidade de eliminar estímulos patogênicos e/ou regular os mecanismos imunitários de supressão leva a uma ativação imune excessiva resultando em um processo inflamatório patológico e perda da tolerância imunológica (Crispe, 2009). Por sua vez, isto pode desencadear em aumento exacerbado de espécies reativas de oxigênio (EROS) tecidual e/ou redução da capacidade de metabolização desses compostos oxidantes, resultando no desequilíbrio do sistema redox, denominado estresse oxidativo. Esta condição é capaz de promover dano em vários tecidos, inclusive no fígado (Jaeschke, 2011). Muitas patologias causadas por protozoários, incluindo Babesia spp e Leishmania spp, helmintos, como Dirofilaria spp., Platynosomum e Clonorchis spp., bactérias, tais como Anaplasma spp., Ehrlichia spp. e Lepstospira spp., e fungos, Histoplasma sp., revelam desordens oxidativas e alterações hepáticas em animais domésticos.

Mediante o exposto, esta revisão visa esclarecer os mecanismos celulares da resposta imune hepática na homeostase e na progressão de distúrbios hepáticos em patologias infecciosas parasitárias de cães e gatos.

\section{Fígado}

O fígado corresponde a um órgão vital de extrema importância uma vez que desempenha funções diretamente envolvidas no controle da homeostase tecidual e sistêmica. Esse desempenho hepático se deve à sua organização estrutural, rede vascular e complexidade celular. O posicionamento do fígado em relação à circulação sanguínea sistêmica é responsável pela dinâmica funcional deste órgão. $\mathrm{O}$ suprimento vascular hepático é oriundo da circulação sanguínea proveniente do trato gastrointestinal e baço via veia porta hepática, formada a partir da confluência de vasos gastroesplênicos e mesentéricos, e do coração via artéria hepática (Crispe, 2009). A veia porta e artéria hepática unem-se no interior do fígado e formam a rede de capilares, denominados sinusóides, que se dispõe em direção as veias centrolobulares, cujas se unem formando as veias hepáticas que drenam o sangue para os demais orgãos pela veia cava (Mccuskey, 2008; Nemeth et al., 2009).

No parênquima hepático, os hepatócitos representam a maior população celular parenquimatosa e estão organizados em fileiras em torno dos sinusóides (Racanelli \& Rehermann, 2006),. Estes são revestidos por células endoteliais fenestradas, conhecidas por células sinusoidais endoteliais, e apresentam em seu interior macrófagos residentes denominados células de Kupffer, células dendríticas e linfócitos. Além dessas, presentes em um espaço estreito existente entre os cordões de hepatócitos e os sinusóides chamado espaço de Disse, ainda há células estreladas (Crispe, 2009; Thomson \& Knolle, 2010). O fígado é o principal órgão envolvido no metabolismo do organismo, intimamente ligado à síntese e distribuição, via circulação sanguínea, de substâncias essenciais ao funcionamento e desempenho dos órgãos extra-hepáticos. As vias metabólicas de degradação e síntese de proteínas, lipídeos e glicídeos ocorrem no interior dos hepatócitos, os quais também estão envolvidos na produção da bile e detoxificação, correspondente a metabolização de drogas, toxinas e espécies reativas de oxigênio, do organismo. Além disso, há o armazenamento de lipídios realizado pelas células estreladas, também produtoras de matriz extracelular (Rui, 2014). Além dessas funções metabólicas essenciais, o 
fígado desempenha uma atividade imunológica complexa mediada por células imunitárias diversificadas e pelas células residentes estruturais. Sendo assim, ele também é capaz de regular a resposta imune.

\section{Microambiente hepático (MH) e a resposta imunológica}

O fígado atua como um órgão da resposta imune inata, uma vez que é exposto facilmente a moléculas patogênicas e patógenos circulantes no sangue portal, proveniente de órgãos linfoides sentinelas e do trato gastrintestinal (Crispe, 2009; Racanelli \& Rehermann, 2006). Além do mais, diversos compostos nocivos derivados de nutrientes ou da microbiota intestinal residente também circulam via veia porta e são capazes de desencadear uma resposta imunológica (Rui, 2014). Apesar de ter essa resposta inata, ele é um órgão que dispõe de um mecanismo de tolerância, em que previne a ativação acidental contra os antígenos nocivos. Portanto, o equilíbrio entre a resposta inflamatória e a tolerância imunológica é importante para manutenção da homeostase. Para exercer essa função, é necessária a participação de uma variedade de células presentes no microambiente hepático (MH) (Crispe, 2009; Racanelli \& Rehermann, 2006).

O MH é constituído por células imunológicas, como células de Kupffer (CK), células dendríticas (CD), linfócitos e populações celulares não hematopoiéticas, tais como hepatócitos, células endoteliais sinusoidais (CES) e células estreladas hepáticas (CEH). Em associação a estas células, participam mediadores como as citocinas próinflamatórias IL-1 (Gehrke et al., 2018), IL-2 (Wittlich et al., 2017), IL-4 e IL-6 (Chi et al., 2018), e as citocinas anti-inflamatórias IL-10 (Krenkel et al., 2014; Liu et al., 2018) e TGF- $\beta$ (Carambia et al., 2014).

Os hepatócitos realizam vários papéis imunológicos, incluindo a produção de proteínas plasmáticas, como fatores de coagulação, complemento e proteínas antimicrobianas; e a produção de proteínas de fase aguda em infecções local e/ou sistêmica (Crispe, 2009). As células de Kupffer são células fagocíticas importantes na remoção de antígenos da circulação hepática, e, assim como as células dendríticas residentes, correspondem às células apresentadoras clássicas (APC) de antígenos. Este papel também pode ser desempenhado pelos hepatócitos, células endoteliais sinusoidais e células estreladas (Thomson \& Knolle, 2010). Essas células produzem citocinas e quimiocinas que regulam o recrutamento e infiltração de leucócitos, tais como neutrófilos, monócitos e células T, bem como modulam a resposta imune e inflamação (Carambia et al., 2014; Heymann et al., 2015; Wehr et al., 2013). Além delas, diversos linfócitos patrulham e residem no tecido hepático, incluindo células $\mathrm{T}$ natural killer (NKT), células natural killer (NK) e, células T CD4+ e CD8+ (Crispe, 2009; Werner et al., 2011; Wittlich et al., 2017).

Em condições fisiológicas normais, padrões moleculares associados aos microrganismos (MAMPs) e/ou padrões moleculares associados a danos (DAMPs), que estão presentes em grandes quantidades no sangue que chega através da veia porta, interagem com os receptores de reconhecimento padrão (PRRs) presentes nas células apresentadoras de antígenos promovendo sua ativação e, consequente, produção de citocinas anti-inflamatórias (Jaeschke, 2011), como a IL-10 (Krenkel et al., 2014; Liu et al., 2018) e TGF- $\beta$ (Carambia et al., 2014). Estas, por sua vez, são capazes de inibir a expressão de moléculas coestimuladoras nas células apresentadoras e ativar células T regulatórias ( $\mathrm{T}$ reg), suprimindo a ativação e expansão de células efetoras, tais como linfócitos T CD4+ (Figura 1) (Carambia et al., 2014).

A resposta imune a MAMPs ou DAMPs resulta em um processo inflamatório controlado por meio da regulação das vias de sinalização pró-inflamatório. Este mecanismo visa proteger o organismo da ativação imune excessiva e dano aos tecidos, influenciando o ambiente imunológico exclusivo dentro do fígado e, então, favorecendo a tolerância (Heymann et al., 2015). Esse estado tolerante é regulado através de fatores desencadeantes, tais como sinalização de receptores do tipo Toll-like (TLR) (Hosein et al., 2015) ou ativação do sistema complemento e inflamassoma, que medeiam à ativação ou supressão das células imunes.

Por sua vez, em situações patológicas, incluindo a infecção por patógenos, pode haver a reversão da tolerância por meio de sinais específicos desses patógenos. A persistência e a falha em eliminar alguns agentes patogênicos, e, portanto, controlar a inflamação, leva à infecção crônica e dano tecidual resultando na perda dos mecanismos de tolerância hepática (Jaeschke, 2011). Em resposta à infecção, CKs ativadas e os hepatócitos danificados liberam mediadores os quais recrutam monócitos e neutrófilos 
inflamatórios para o fígado. Estes fagócitos ativados produzem citocinas reguladoras como IL-1 e IL-6 (Chi et al., 2018; Gehrke et al., 2018), as quais promovem a infiltração e ativação de várias populações linfocitárias efetoras bem como agem diretamente sobre os hepatócitos íntegros induzindo à produção de proteínas de fase aguda e a reparação tecidual. Ao mesmo tempo, no sítio infeccioso, os macrófagos e neutrófilos interagem com os patógenos a fim de eliminá-los pelo processo de fagocitose associado à produção de óxido nítrico e espécies reativas de oxigênio (EROs) (Figura 1).

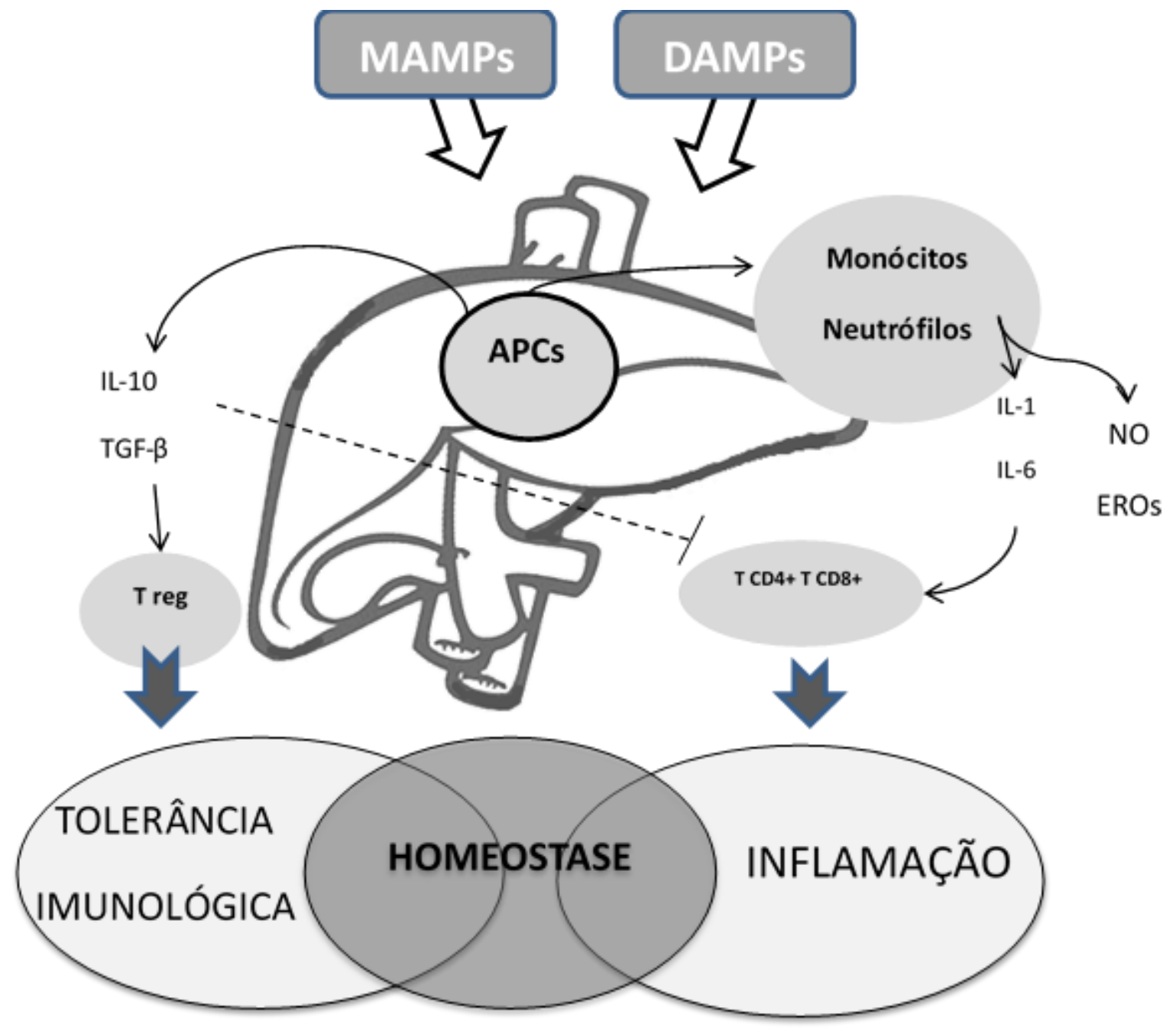

Figura 1. Representação esquemática do mecanismo imune hepático na manutenção da homeostase e a modulação para condições de tolerância ou inflamação. MAMPs: padrões moleculares associados aos microrganismos; DAMPs: padrões moleculares associados a danos; APCs: células apresentadoras clássicas de antígenos; IL-10 e TGF- $\beta$ : citocinas antiinflamatórias; IL-1 e IL-6: citocinas próinflamatórias; T reg: células T regulatórias; T CD4+ e T CD8+: células T efetoras CD4+ e CD8+; NO: óxido nítrico; EROs: espécies reativas de oxigênio.

Em detrimento da exacerbação e/ou desregulação da inflamação pelos patógenos pode ocorrer um aumento de ERO decorrente da produção elevada pelas células fagocíticas e prejuízo metabólico desses compostos oxidantes pelos hepatócitos danificados, resultando no desequilíbrio entre os agentes oxidantes e antioxidantes, denominado estresse oxidativo. No fígado, os oxidantes podem causar danos às células parenquimatosas, os hepatócitos (Jaeschke, 2011), levando a prejuízo no metabolismo hepático e, consequentemente, comprometimento de órgãos extra-hepáticos. Além dessas, as células de Kupffer, células estreladas hepáticas e células endoteliais sinusoidais também são sensíveis e, potencialmente, expostas a moléculas relacionadas ao estresse oxidativo. Este induz a produção de uma variedade de citocinas como o TNF- $\alpha$, que pode exacerbar a inflamação e a apoptose. Por outro lado, a peroxidação lipídica pode desencadear aumento na proliferação e síntese de colágeno pelas células estreladas hepáticas (Sakaguchi et al., 2010; Sánchez-Valle et al., 2012). Portanto, o estresse oxidativo desempenha um papel crítico no desenvolvimento de distúrbios e patologias hepáticas. 


\section{O estresse oxidativo nas patologias infecto-parasitárias de caninos e felinos}

Em diferentes doenças infecciosas parasitárias nos animais domésticos, incluindo os caninos e felinos (Rubio et al., 2017; Vilhena et al., 2017), têm sido relatado à participação de desordens oxidativas (Tabela 1). A maioria das condições patológicas revela uma desregulação nos mecanismos de oxirredução, favorecendo um desequilíbrio na produção dos radicais livres e/ou defeito no sistema de defesa antioxidante, ou seja, o estado de estresse oxidativo. Os radicais livres denominados espécies reativas de oxigênio (EROs), como o ânion superóxido $\left(\mathrm{O}_{2}^{-}\right)$e a hidroxila $\left(\mathrm{OH}^{\circ}\right)$, e espécies reativas de nitrogênio (ERN), tais como o peroxinitro $\left(\mathrm{ONOO}^{-}\right)$, o óxido nitroso $\left(\mathrm{N}_{2} \mathrm{O}_{3}\right)$, nitritos $\left(\mathrm{NO}_{2}{ }^{-}\right)$e nitratos $\left(\mathrm{NO}_{3}^{-}\right)$, são produzidos em detrimento de vários processos fisiológicos naturais do organismo e em decorrência de disfunções biológicas ou patológicas (Barbosa et al., 2010; Jaeschke, 2011; Oliveira \& Schoffen, 2010). Logo, o aumento descontrolado no nível desses agentes oxidantes, EROs e ERNs, podem promover ações deletérias no organismo, tais como: ruptura da membrana celular, mutação do DNA, oxidação dos lipídios insaturados e o comprometimento dos componentes da matriz extracelular (Barreiros et al., 2006).

Tabela 1. Biomarcadores do estresse oxidativo e as alterações hepáticas associados à agentes parasitários em cães e gatos.

\begin{tabular}{|c|c|c|c|}
\hline Parasito & Biomarcadores & Alterações hepáticas & Referências \\
\hline \multirow{6}{*}{ Ehrlichia canis } & \multirow{6}{*}{$\begin{array}{l}\uparrow \mathrm{AOPP}^{1}, \mathrm{NO}^{2}, \mathrm{MDA}^{2}, \mathrm{PCR}^{3,4} \mathrm{e} \\
\mathrm{GR}^{2} ; \\
\downarrow \mathrm{EAT}^{5}, \mathrm{Alb}^{3,4} \text { e PON-1 }{ }^{3,4}\end{array}$} & \multirow{6}{*}{ Infiltrado inflamatório $^{18}$, granuloma ${ }^{18}$} & ${ }^{1}$ Bottari et al. (2016) \\
\hline & & & ${ }^{2}$ Silva et al. (2013) \\
\hline & & & ${ }^{3}$ Karnezi et al. (2016) \\
\hline & & & ${ }^{4}$ Rubio et al. (2017) \\
\hline & & & ${ }^{5}$ Rudoler et al. (2015) \\
\hline & & & ${ }^{18}$ Nair et al. (2016) \\
\hline \multirow{2}{*}{ Babesia canis } & $\uparrow \mathrm{MDA}^{6}$ e $\mathrm{Bil}^{6}$ & \multirow{2}{*}{ Hepatomegalia $^{19} ; \uparrow A L T$ e FA ${ }^{19}$} & ${ }^{6}$ Crnogaj et al. (2017) \\
\hline & $\downarrow \mathrm{SOD}^{6}, \mathrm{CAT}^{6} \mathrm{e} \mathrm{GPx}^{6}$ & & ${ }^{19}$ Fraga et al. (2011) \\
\hline Babesia vogeli & $\downarrow \mathrm{PON}-1^{7}$ & n.d. & ${ }^{7}$ Vilhena et al. (2017) \\
\hline \multirow{12}{*}{ Leishmania spp. } & \multirow{12}{*}{$\begin{array}{l}\uparrow \mathrm{MDA}^{8,9} \text { e PCR }{ }^{10} \\
\downarrow \mathrm{SOD}^{11,12}, \mathrm{CAT}^{11,12}, \mathrm{GPx}^{11,12} \\
\mathrm{EAT}^{13}, \mathrm{Acu}^{13} \text { e } \mathrm{Alb}^{13,14,15}\end{array}$} & \multirow{12}{*}{$\begin{array}{l}\text { Infiltrado inflamatório }{ }^{12,20,} \text {, degeneração } \\
\text { de hepatócitos }{ }^{12,20} \text {, hepatomegalia }{ }^{12,15,21} \text {, } \\
\text { congestão }^{12,15,21} \text {, granuloma }{ }^{12,15,21} \mathrm{e} \\
\text { fibrose }^{22 \mathrm{e}} 23 ; \uparrow \mathrm{ALT} \text { e } \mathrm{FA}^{20}\end{array}$} & ${ }^{8}$ Bildik et al. (2004) \\
\hline & & & ${ }^{9}$ Santos et al. (2016) \\
\hline & & & ${ }^{10}$ Lopes-Neto et al. (2015) \\
\hline & & & ${ }^{11}$ Lopes-Neto et al. (2016) \\
\hline & & & ${ }^{12}$ Souza et al. (2014) \\
\hline & & & ${ }^{13}$ Almeida et al. (2013) \\
\hline & & & ${ }^{14}$ Freitas et al. (2012) \\
\hline & & & ${ }^{15}$ Giunchetti et al. (2008) \\
\hline & & & ${ }^{20}$ Torres et al. (2016) \\
\hline & & & ${ }^{21}$ Castro et al. (2018) \\
\hline & & & ${ }^{22}$ Melo et al. (2009) \\
\hline & & & ${ }^{23}$ Madeira et al. (2016) \\
\hline \multirow{3}{*}{ Dirofilaria immitis } & \multirow{3}{*}{$\begin{array}{l}\uparrow \mathrm{PCR}^{16,17} ; \\
\downarrow \text { Alb e PON-1 } 1^{16,17}\end{array}$} & \multirow{3}{*}{ Fibrose hepática e congestão ${ }^{24}$} & ${ }^{16}$ Méndez et al. (2014) \\
\hline & & & ${ }^{17}$ Carretón et al. (2017) \\
\hline & & & ${ }^{24}$ Chikweto et al. (2014) \\
\hline
\end{tabular}

AOPP: produtos de proteínas de oxidação; NO: óxido nítrico; MDA: malondialdeido; PON-1: paraoxonase-1; SOD: superóxido dismutase; CAT: catalase; GPx: glutationa peroxidase; GR: glutationa redutase; EAT: estado antioxidante total; PCR: proteína C reativa; Bil: bilirrubina; Acu: ácido úrico; Alb: albumina; ALT: alanina aminotransferase; FA: fosfatase alcalina. $\uparrow:$ aumento; $\downarrow$ :diminuição; n.d.: não determinado. Fonte: Autores (2019).

$\mathrm{Na}$ erliquiose canina, doença causada pela bactéria Ehrlichia canis, pode ser observado aumento significativo nos níveis de EROs (Rubio et al., 2017), incluindo produtos oxidativos AOPP (produtos de proteínas de oxidação) (Bottari et al., 2016) e óxido nítrico (NO*) (Silva et al., 2013). Este corresponde a um composto produzido por ação da enzima óxido nítrico sintetase induzida (iNOS) no interior de fagócitos a fim de debelar os patógenos, uma vez que a partir dele são formadas substâncias tóxicas deletérias, as ERNs (Barreiros et al., 2006). Além disso, há evidências de aumento nas concentrações de malondialdeído (MDA) (Silva et al., 2013), achado semelhante na babesiose (Crnogaj et al., 2017) e leishmaniose canina (Bildik et al., 2004; Santos et al., 2016), doenças transmitidas por 
vetores e causadas por protozoários. Portanto, o aumento de compostos oxidativos levam ao processo de lipoperoxidação, representado pelo aumento de MDA.

Na infecção por patógenos, o aumento de EROS ocorre em virtude da resposta imune inflamatória, diretamente relacionada a alterações nas proteínas de fase aguda (APP) tais como proteína $\mathrm{C}$ reativa (PCR), albumina e paraoxonase-1 (PON-1). Essas proteínas são produzidas pelo hepatócitos modulando a resposta sistêmica (Mackness et al., 2010; Moshage, 1997).

Na dirofilariose canina, doença crônica transmitida por vetores e causada pelo nematódeo Dirofilaria immitis, foi evidenciado aumento nas concentrações de PCR e diminuição nos níveis de albumina (Carretón et al., 2017; Méndez et al., 2014), achado semelhante foi evidenciado na erliquiose (Karnezi et al., 2016; Rubio et al., 2017) e leishmaniose canina (Almeida et al., 2013; Freitas et al., 2012; Giunchetti et al., 2008; Lopes-Neto et al., 2015). Além disso, relataram diminuição nos níveis de PON1 (Carretón et al., 2017; Méndez et al., 2014), assim como na babesiose felina por Babesia vogeli (Vilhena et al., 2017). Sendo assim, esses achados refletem a ligação entre o processo inflamatório e o dano oxidativo. Por outro lado, o organismo dispõe de um sistema antioxidante a fim de controlar as concentrações e ações dos radicais livres, mantendo em baixas concentrações as espécies reativas ao oxigênio (EROs) nas células e tecidos biológicos e reparando estruturas danificadas. Esse sistema é compreendido por antioxidantes enzimáticos, como a glutationa peroxidase (GPx), glutationa redutase (GR), superóxido dismutase (SOD) e catalase (CAT) e compostos não enzimáticos, tais como glutationa (GSH), ácido úrico e bilirrubina (Barreiros et al., 2006; Oliveira \& Schoffen, 2010; Świderska et al., 2019).

Estudos realizados em cães infectados por Babesia canis e Leishmania infamtum revelaram redução da atividade antioxidante sérica das enzimas SOD, CAT e GPx (Crnogaj et al., 2017; Lopes-Neto et al., 2016; Souza et al., 2014). Enquanto isso, na erliquiose canina, foi relatado aumento na atividade antioxidante da GR (Silva et al., 2013) e redução no estado antioxidante total (EAT) dos animais infectados (Rudoler et al., 2015). Este último dado também foi verificado na leishmaniose canina (Almeida et al., 2013). Em resumo, isso poderia explicar a redução na capacidade antioxidante diante do consumo dos antioxidantes para catalizar os radicais livres que foram produzidos em excesso durante a resposta imune inflamatória ao patógeno.

Os antioxidantes que também têm suas atividades reconhecidas são os não enzimáticos como a bilirrubinae o ácido úrico. Na babesiose canina, causada por Babesia canis, também foi vista alteração nos níveis de bilirrubina (Crnogaj et al., 2017), que atua como um antioxidante em interação com a albumina sérica, sendo esse complexo considerado um dos antioxidantes naturais dos fluidos extracelulares (Barreiros et al., 2006). Por outro lado, na leishmaniose canina foi relatada redução do ácido úrico, que corresponde a um substrato antioxidante plasmático (Almeida et al., 2013).

\section{Resposta hepática aos agentes infecciosos}

Alterações hepáticas têm sido vistas em patologias associadas à agentes infecciosos de origens diversas, incluindo protozoários, helmintos, bactérias, fungos entre outros (Tabela 1). A maioria das moléculas patogênicas e patógenos infecciosos circulantes, oriundos de órgãos linfoides periféricos e do trato gastrintestinal, via circulação sanguínea, permeia pelo tecido hepático e são capazes de desencadear uma resposta imune exacerbada de modo a romper a tolerância imunológica. Por sua vez, a desregulação imunológica favorece o estresse oxidativo. Diante disso, o fígado, enriquecido por células imune inata, compreende um órgão central da resposta imunológica à agentes infecciosos.

A Ehrlichia canis e o Anaplasma phagocytophilum correspondem a bactérias intracelulares de leucócitos mononucleares, transmitidas aos cães pelos carrapatos. Nas células infectadas, esses microorganismos se multiplicam e, então, são liberados por citólise para infectar outras células. Os fagócitos parasitados e os patógenos livres margeiam os vasos sanguíneos atingindo o fígado, e promovem a estimulação do sistema imune hepático, composto por uma grande população de macrófagos residentes, CKs (Chirek et al., 2018; Nair et al., 2016). Babesia canis compreende um protozoário, transmitido também através de carrapatos aos cães, que se replica no interior dos eritrócitos promovendo lise celular, resultando em anemia hemolítica. Esse parasitismo induz uma resposta imunomediada, estimulando a ativação e proliferação de células inflamatórias, e, por sua vez, 
potencializando a anemia e a resposta dos órgãos imunes (Fraga et al., 2011), inclusive fígado e baço. Histoplasma capsulatum é um fungo patogênico que apresenta afinidade pelo sistema reticuloendotelial, assim como a E. canis e o A. phagocytophilum, circulando por todo corpo através do sangue e da linfa no interior de fagócitos mononucleares, atingindo múltiplos orgãos, inclusive o fígado (Klang et al., 2013). Na infecção por E. canis e E. chaffeensis, as alterações hepáticas envolvem a presença de infiltrados de macrófagos e linfócitos perivascular com microgranulomas sinusoidais e periportais (Nair et al., 2016). Em cães acometidos por B. canis (Fraga et al., 2011) e A. phagocytophilum (Nair et al., 2016), podem ser observados hepatomegalia, achado semelhante em gato infectado por $H$. capsulatum (Klang et al., 2013).

Platynossomun spp. é um helminto trematóide que apresenta predileção pelo trato biliar, por outro lado migra facilmente para o fígado ativando o complexo imune hepático (Braga et al., 2016). Dirofilaria immitis corresponde a pequenos helmintos, conhecidos como microfilárias, que apresentam predileção pelas artérias pulmonares. Em processos infecciosos persistentes, os vermes adultos podem seguir por um fluxo retrógrado atingindo a veia cava (Chikweto et al., 2014). Lepstospira interrogans corresponde a uma bactéria espiroqueta, que circula pelo organismo podendo atingir e promover alterações em diversos órgãos, destacando-se o fígado. A alteração hepática é decorrente da resposta inflamatória tecidual à replicação do patógeno, bem como a produção de toxinas (Larson et al., 2017).

Na platinossomíase em gatos domésticos, causada pelo trematóide Platynosomum fastosum, também são observados infiltrados inflamatórios caracterizados por macrófagos, linfócitos e plasmócitos. Além disso, pode ser evidenciada fibrose hepática (Braga et al., 2016), achado semelhante na infecção de cães por $D$. immmitis resultante da obstrução parasitária que leva a congestão e à síndrome da veia cava (Chikweto et al., 2014). Lesões ulcerativas necróticas são vistas na platinossomíase bem como na leptospirose canina causada por Lepstospira interrogans sorovar Copenhageni (Larson et al., 2017). Por outro lado, a dilatação em ducto biliar intrahepático foi encontrada em cães infectados por Clonorchis sinensis, que é um helminto com alto risco de patogenicidade (Ye et al., 2013).

A leishmaniose é uma doença multifatorial, causada por protozoários intracelulares obrigatórios Leishmania spp e transmitida por meio de vetores flebotomíneos aos cães. As células mononucleares infectadas migram pela circulação sanguínea atingindo diversos órgãos como o baço, linfonodos e tecidos como pele e intestino, além do tecido hematopoético, estimulando o sistema imune de forma localizada e sistêmica. Neste sentido, L. infantum circula pelo tecido hepático e induz infiltrado inflamatório por decorrente de fatores quimiotáticos liberados pelas CKs parasitadas (Rodrigues et al., 2019) e CEH, causando hepatite periportal e degeneração de hepatócitos (Souza et al., 2014; Torres et al., 2016). Em resposta a infecção, o fígado torna-se aumentado (hepatomegalia) e congesto associado à reação inflamatória granulomatosa crônica, caracterizada por numerosos granulomas hepáticos intralobulares (Giunchetti et al., 2008; Souza et al., 2014). Este processo inflamatório granulomatoso leva à fibropoiese hepática intensa nos cães naturalmente infectados com L. (Leishmania) infantum (Melo et al., 2009). O aumento na deposição de colágeno tecidual se deve a maior expressão de TGF- $\beta$ pelas células do granuloma e, então, ativação das CEH (Castro et al., 2018; Madeira et al., 2016).

Na babesiose, anaplasmose e leishmaniose canina, além das alterações histológicas, foram relatados aumentos nas concentrações séricas das enzimas hepáticas alanina aminotransferase (ALT) e fosfatase alcalina (FA) (Chirek et al., 2018; Fraga et al., 2011; Torres et al., 2016). Essas enzimas estão presentes no citoplasma e membrana dos hepatócitos podendo, então, indicar lesão celular uma vez que elas estão alteradas. Na leishmaniose pode-se observar também diminuição nos níveis séricos de fibrinogênio (Torres et al., 2016), provavelmente associado também ao dano aos hepatócitos uma vez que são responsáveis pela produção desse fator de coagulação.

\section{Considerações finais}

A maioria dos agentes infecciosos parasitários desencadeia o aparecimento de alterações no fígado, bem como promovem um desequilíbrio no sistema redox resultando no estresse oxidativo. Diante disso, vimos que as complicações histológicas do fígado são consequências da resposta inata aos patógenos com indução do recrutamento de várias células imunes inflamatórias que promovem alteração da função hepática, inclusive em decorrência do estado de estresse oxidativo. Portanto, concluímos que o fígado é um importante órgão de defesa nas patologias parasitárias de animais domésticos. 


\section{Referências bibliográficas}

Almeida, B. F. M., Narciso, L. G., Melo, L. M., Preve, P. P., Bosco, A. M., Lima, V. M. F. \& Ciarlini, P. C. (2013). Leishmaniasis causes oxidative stress and alteration of oxidative metabolism and viability of neutrophils in dogs. The Veterinary Journal, 198(3):599-605.

Barbosa, K. B. F., Costa, N. M. B., Alfenas, R. d. C. G., De Paula, S. O., Minim, V. P. R. \& Bressan, J. (2010). Estresse oxidativo: conceito, implicações e fatores modulatórios. Revista de Nutrição, 23(4):629-643.

Barreiros, A. L. B. S., David, J. M. \& David, J. P. (2006). Estresse oxidativo: relação entre geração de espécies reativas e defesa do organismo. Química Nova, 29(1):113-123.

Bildik, A., Kargın, F., Seyrek, K., Pasa, S. \& Özensoy, S. (2004). Oxidative stress and non-enzymatic antioxidative status in dogs with visceral Leishmaniasis. Research in Veterinary Science, 77(1):6366.

Bottari, N. B., Crivellenti, L. Z., Borin-Crivellenti, S., Oliveira, J. R., Coelho, S. B., Contin, C. M., . . . Tonin, A. A. (2016). Iron metabolism and oxidative profile of dogs naturally infected by Ehrlichia canis: Acute and subclinical disease. Microbial Pathogenesis, 9226-29.

Braga, R. R., Teixeira, A. C., Oliveira, J. A. A. \& Cavalcanti, L. P. G. (2016). Prevalence of Platynosomum fastosum infection in free roaming cats in northeastern Brazil: Fluke burden and grading of lesions. Veterinary Parasitology, 22720-25.

Carambia, A., Freund, B., Schwinge, D., Heine, M., Laschtowitz, A., Huber, S., . . Lohse, A. W. (2014). TGF- $\beta$-dependent induction of CD4+ CD25+ Foxp3+ Tregs by liver sinusoidal endothelial cells. Journal of Hepatology, 61(3):594-599.

Carretón, E., Cerón, J. J., Martínez-Subiela, S., Tvarijonaviciute, A., Caro-Vadillo, A. \& MontoyaAlonso, J. A. (2017). Acute phase proteins and markers of oxidative stress to assess the severity of the pulmonary hypertension in heartworm-infected dogs. Parasites \& Vectors, 10(2):1-9.

Castro, R. S., Amorim, I. F. G., Pereira, R. A., Silva, S. M., Pinheiro, L. J., Pinto, A. J. W., . . Mosser, D. M. (2018). Hepatic fibropoiesis in dogs naturally infected with Leishmania (Leishmania) infantum treated with liposome-encapsulated meglumine antimoniate and allopurinol. Veterinary Parasitology, 25022-29.

Chi, G., Feng, X.-X., Ru, Y.-X., Xiong, T., Gao, Y., Wang, H., . . He, Y.-P. (2018). TLR2/4 ligandamplified liver inflammation promotes initiation of autoimmune hepatitis due to sustained IL-6/IL12/IL-4/IL-25 expression. Molecular Immunology, 99171-181.

Chikweto, A., Bhaiyat, M. I., Lanza-Perea, M., Veytsman, S., Tiwari, K., Allie, C. \& Sharma, R. N. (2014). Retrospective study of canine heartworm disease with caval syndrome in Grenada, West Indies. Veterinary Parasitology, 205(3-4):721-724.

Chirek, A., Silaghi, C., Pfister, K. \& Kohn, B. (2018). Granulocytic anaplasmosis in 63 dogs: clinical signs, laboratory results, therapy and course of disease. Journal of Small Animal Practice, 59(2):112120.

Crispe, I. N. (2009). The liver as a lymphoid organ. Annual Review of Immunology, 27147-163.

Crnogaj, M., Cerón, J. J., Šmit, I., Kiš, I., Gotić, J., Brkljačić, M. \& Mrljak, V. (2017). Relation of antioxidant status at admission and disease severity and outcome in dogs naturally infected with Babesia canis canis. BMC Veterinary Research, 13(1):1-9.

Fraga, E., Barreiro, J. D., Goicoa, A., Espino, L., Fraga, G. \& Barreiro, A. (2011). Abdominal ultrasonographic findings in dogs naturally infected with babesiosis. Veterinary Radiology \& Ultrasound, 52(3):323-329.

Freitas, J. C. C., Nunes-Pinheiro, D. C. S., Lopes Neto, B. E., Santos, G. J. L., Abreu, C. R. A., Braga, R. R., . . Oliveira, L. F. (2012). Clinical and laboratory alterations in dogs naturally infected by Leishmania chagasi. Revista da Sociedade Brasileira de Medicina Tropical, 45(1):24-29.

Gehrke, N., Hövelmeyer, N., Waisman, A., Straub, B. K., Weinmann-Menke, J., Wörns, M. A., . . . Schattenberg, J. M. (2018). Hepatocyte-specific deletion of IL1-RI attenuates liver injury by blocking IL-1 driven autoinflammation. Journal of Hepatology, 68(5):986-995. 
Giunchetti, R. C., Mayrink, W., Carneiro, C. M., Corrêa-Oliveira, R., Martins-Filho, O. A., Marques, M. J. \& Reis, A. B. (2008). Histopathological and immunohistochemical investigations of the hepatic compartment associated with parasitism and serum biochemical changes in canine visceral leishmaniasis. Research in Veterinary Science, 84(2):269-277.

Heymann, F., Peusquens, J., Ludwig-Portugall, I., Kohlhepp, M., Ergen, C., Niemietz, P., . . Randolph, G. J. (2015). Liver inflammation abrogates immunological tolerance induced by Kupffer cells. Hepatology, 62(1):279-291.

Hosein, S., Rodríguez-Cortés, A., Blake, D. P., Allenspach, K., Alberola, J. \& Solano-Gallego, L. (2015). Transcription of toll-like receptors 2, 3, 4 and 9, FoxP3 and Th17 cytokines in a susceptible experimental model of canine Leishmania infantum infection. PloS one, 10(10):e0140325.

Jaeschke, H. (2011). Reactive oxygen and mechanisms of inflammatory liver injury: present concepts. Journal of Gastroenterology and Hepatology, 26173-179.

Karnezi, D., Ceron, J. J., Theodorou, K., Leontides, L., Siarkou, V. I., Martinez, S., . . Pardali, D. (2016). Acute phase protein and antioxidant responses in dogs with experimental acute monocytic ehrlichiosis treated with rifampicin. Veterinary Microbiology, 18459-63.

Klang, A., Loncaric, I., Spergser, J., Eigelsreiter, S. \& Weissenböck, H. (2013). Disseminated histoplasmosis in a domestic cat imported from the USA to Austria. Medical Mycology Case Reports, 2108-112.

Krenkel, O., Mossanen, J. C. \& Tacke, F. (2014). Immune mechanisms in acetaminophen-induced acute liver failure. Hepatobiliary Surgery and Nutrition, 3(6):331-343.

Larson, C. R., Dennis, M., Nair, R. V., Llanes, A., Peda, A., Welcome, S. \& Rajeev, S. (2017). Isolation and characterization of Leptospira interrogans serovar Copenhageni from a dog from Saint Kitts. JMM Case Reports, 4(10):e005120.

Liu, J., Yu, Q., Wu, W., Huang, X., Broering, R., Werner, M., . . Lu, M. (2018). TLR2 stimulation strengthens intrahepatic myeloid-derived cell-mediated T cell tolerance through inducing Kupffer cell expansion and IL-10 production. The Journal of Immunology, 200(7):2341-2351.

Lopes-Neto, B. E., Lima, A. L., Santos, G. J. L., Gonçalves, L. D., Maia, F. M. M., Freitas, J. C. C. \& Nunes-Pinheiro, D. C. S. (2015). Avaliação da resposta inflamatória em cães naturalmente infectados por Leishmania infantum. Revista Brasileira de Higiene e Sanidade Animal, 9(4):645-656.

Lopes-Neto, B. E., Santos, G. J. L., Lima, A. L., Barbosa, M. C., Santos, T. E. J., Uchoa, D. C., . . . Nunes-Pinheiro, D. C. S. (2016). Catalase and glutathione peroxidase in dogs naturally infected by Leishmania infantum. Acta Scientiae Veterinariae, 441-6.

Mackness, B., Beltran-Debon, R., Aragones, G., Joven, J., Camps, J. \& Mackness, M. (2010). Human tissue distribution of paraoxonases 1 and 2 mRNA. IUBMB Life, 62(6):480-482.

Madeira, I. M. V. M., Pereira, D. M. O., Sousa, A. A., Vilela, C. A., Amorim, I. F. G., Caliari, M. V., Tafuri, W. L. (2016). Immunohistochemical study of hepatic fibropoiesis associated with canine visceral leishmaniasis. International Journal of Experimental Pathology, 97(2):139-149.

Mccuskey, R. S. (2008). The hepatic microvascular system in health and its response to toxicants. The Anatomical Record, 291: 661-671.

Melo, F. A., Moura, E. P., Ribeiro, R. R., Alves, C. F., Caliari, M. V., Tafuri, W. L., . . Tafuri, W. L. (2009). Hepatic extracellular matrix alterations in dogs naturally infected with Leishmania (Leishmania) chagasi. International Journal of Experimental Pathology, 90(5):538-548.

Méndez, J. C., Carretón, E., Martínez, S., Tvarijonaviciute, A., Cerón, J. J. \& Montoya-Alonso, J. A. (2014). Acute phase response in dogs with Dirofilaria immitis. Veterinary Parasitology, 204(34):420-425.

Moshage, H. (1997). Cytokines and the hepatic acute phase response. The Journal of Pathology, 181(3):257-266.

Nair, A. D. S., Cheng, C., Ganta, C. K., Sanderson, M. W., Alleman, A. R., Munderloh, U. G. \& Ganta, R. R. (2016). Comparative experimental infection study in dogs with Ehrlichia canis, E. chaffeensis, Anaplasma platys and A. phagocytophilum. PLoS One, 11(2):e0148239. 
Nemeth, E., Baird, A. W. \& O'Farrelly, C. (2009). Microanatomy of the liver immune system. Paper presented at the Seminars in Immunopathology.

Oliveira, M. C. d. \& Schoffen, J. P. F. (2010). Oxidative stress action in cellular aging. Brazilian Archives of Biology and Technology, 53(6):1333-1342.

Racanelli, V. \& Rehermann, B. (2006). The liver as an immunological organ. Hepatology, 43(S1):S54S62.

Rodrigues, A., Alexandre-Pires, G., Valério-Bolas, A., Santos-Mateus, D., Rafael-Fernandes, M., Pereira, M. A. \& Lopes-Ventura, S. (2019). Dog hepatocytes are key effector cells in the liver innate immune response to Leishmania infantum. Parasitology, 146(6):753-764.

Rubio, C. P., Yilmaz, Z., Martínez-Subiela, S., Kocaturk, M., Hernández-Ruiz, J., Yalcin, E., . . Ceron, J. J. (2017). Serum antioxidant capacity and oxidative damage in clinical and subclinical canine ehrlichiosis. Research in Veterinary Science, 115301-306.

Rudoler, N., Harrus, S., Martinez-Subiela, S., Tvarijonaviciute, A., van Straten, M., Cerón, J. J. \& Baneth, G. (2015). Comparison of the acute phase protein and antioxidant responses in dogs vaccinated against canine monocytic ehrlichiosis and naive-challenged dogs. Parasites \& Vectors, $8(1): 175$.

Rui, L. (2014). Energy metabolism in the liver. Comprehensive Physiology, 4(1):117-197.

Sakaguchi, S., Takahashi, S., Sasaki, T., Kumagai, T. \& Nagata, K. (2010). Progression of alcoholic or non-alcoholic steatohepatitis; common metabolic aspects of innate immune system and oxidative stress. Drug Metabolism and Pharmacokinetics, 2630-46.

Sánchez-Valle, V., Chavez-Tapia, N. C., Uribe, M. \& Méndez-Sánchez, N. (2012). Role of oxidative stress and molecular changes in liver fibrosis: a review. Current Medicinal Chemistry, 19(28):48504860.

Santos, G. J. L., Lima, A. L., Lopes-Neto, B. E., Freitas, J. C. C., Maia, F. M. M. \& Nunes-Pinheiro, D. C. S. (2016). Adesão leucocitária e MDA sérico em cães naturalmente infectados por Leishmania infantum. Revista Brasileira de Ciência Veterinária, 23(1-2):48-54.

Silva, A. S., Munhoz, T. D., Faria, J. L. M., Vargas-Hérnandez, G., Machado, R. Z., Almeida, T. C., . . . Tinucci-Costa, M. (2013). Increase nitric oxide and oxidative stress in dogs experimentally infected by Ehrlichia canis: effect on the pathogenesis of the disease. Veterinary Microbiology, 164(3-4):366369.

Souza, C. C., Barreto, T. O., Silva, S. M., Pinto, A. W. J., Figueiredo, M. M., Rocha, O. G. F., .. Tafuri, W. L. (2014). A potential link among antioxidant enzymes, histopathology and trace elements in canine visceral leishmaniasis. International Journal of Experimental Pathology, 95(4):260-270.

Świderska, M., Maciejczyk, M., Zalewska, A., Pogorzelska, J., Flisiak, R. \& Chabowski, A. (2019). Oxidative stress biomarkers in the serum and plasma of patients with nonalcoholic fatty liver disease (NAFLD). Can plasma AGE be a marker of NAFLD? Oxidative stress biomarkers in NAFLD patients. Free Radical Research1-11.

Thomson, A. W. \& Knolle, P. A. (2010). Antigen-presenting cell function in the tolerogenic liver environment. Nature Reviews Immunology, 10(11):753-766.

Torres, M. M., Almeida, A. B. P. F., Paula, D. A. J., Mendonça, A. J., Nakazato, L., Pescador, C. A. \& Sousa, V. R. F. (2016). Hemostatic assessment of dogs associated with hepatic parasite load of Leishmania infantum chagasi. Revista Brasileira de Parasitologia Veterinária, 25(2):244-247.

Vilhena, H., Tvarijonaviciute, A., Cerón, J. J., Vieira, L., Pastor, J. \& Silvestre-Ferreira, A. C. (2017). Acute phase proteins response in cats naturally infected with Hepatozoon felis and Babesia vogeli. Veterinary Clinical Pathology, 46(1):72-76.

Wehr, A., Baeck, C., Heymann, F., Niemietz, P. M., Hammerich, L., Martin, C., . . Hittatiya, K. (2013). Chemokine receptor CXCR6-dependent hepatic NK T Cell accumulation promotes inflammation and liver fibrosis. The Journal of Immunology, 190(10):5226-5236.

Werner, J. M., Lang, C., Scherer, M. N., Farkas, S. A., Geissler, E. K., Schlitt, H. J. \& Hornung, M. (2011). Distribution of intrahepatic T, NK and CD3+ CD56+ NKT cells alters after liver transplantation: Shift from innate to adaptive immunity? Transplant Immunology, 25(1):27-33. 
Wittlich, M., Dudek, M., Böttcher, J. P., Schanz, O., Hegenbarth, S., Bopp, T. \& John, S. R. (2017). Liver sinusoidal endothelial cell cross-priming is supported by CD4 T cell-derived IL-2. Journal of Hepatology, 66(5):978-986.

Ye, C.-Y., Yang, Z.-D. \& Zheng, H.-L. (2013). Fatal multi-organ Clonorchis sinensis infection in dog: A case report. Veterinary Parasitology, 195(1-2):173-176.

Recebido: 18 de julho, 2019.

Aprovado: 17 de agosto, 2019.

Publicado: 10 de outubro, 2019.

Licenciamento: Este artigo é publicado na modalidade Acesso Aberto sob a licença Creative Commons Atribuição 4.0 (CC-BY 4.0), a qual permite uso irrestrito, distribuição, reprodução em qualquer meio, desde que $\mathrm{o}$ autor e a fonte sejam devidamente creditados. 